\title{
Parir y nacer en tiempos de covID-19 en Uruguay*
}

\author{
Delivery and birth during CovID-19 in Uruguay
}

\author{
MARIANA VIERA** Y VICTORIA EVIA***
}

\begin{abstract}
The purpose of the article is to analyze the structural dimensions of the social production of delivery and birth in the context of COVID-19 in Uruguay, focusing on women's experiences, based on the dialogue between critical medical anthropology and feminist anthropology. Through a qualitative and exploratory methodology, secondary data are linked to the interviews of eightwomen of medium-high socio-educational level. We conclude that their experiences, care trajectories and strategies deployed, were crossed by fears and uncertainties regarding respect for their enshrined rights during the care of pregnancy, childbirth and birth in the health services by appealing to arguments of exceptionality. This article contributes to the understanding of the cases' continuities and exceptionalities, taking into account the variables of gender, class and nationality. Additionally, this article also contributes to the fruitful development of dialogues between both conceptual approaches.
\end{abstract}

Key words: Critical Medical Anthropology, birth, risk, uncertainty, reproductive rights, gender, feminism

\section{Resumen}

El objetivo de este artículo es analizar las dimensiones estructurales de la producción social del parto y el nacimiento en el contexto de la COVID-19 en Uruguay a partir del diálogo entre la antropología médica crítica y la antropología feminista, poniendo en el centro las experiencias de mujeres. Mediante una metodología cualitativa y exploratoria se relacionan datos secundarios con los relatos de ocho mujeres de nivel socioeducativo medio-alto. Se concluye que sus experiencias, trayectorias de atención y estrategias desplegadas estuvieron marcadas por los temores e incertidumbres en cuanto al respeto de los derechos consagrados durante la atención al embarazo, el parto y el nacimiento en los servicios de salud, pues estos últimos apelaban al argumento de excepcionalidad. Se aporta a la comprensión de continuidades y excepcionalidades del caso, considerando las variables de género, clase y nacionalidad, así como al fructífero desarrollo de diálogos entre la antropología médica crítica y la antropología feminista.

Palabras clave: antropología médica crítica, riesgo, incertidumbre, género, derechos reproductivos, feminismo

\footnotetext{
* Artículo recibido el 02/10/20 y aceptado el 04/01/21.

** Universidad de la República, Uruguay. Av. 18 de Julio 1824, 11200, Montevideo, Departamento de Montevideo, Uruguay <marianavieracherro@gmail.com>.

*** Universidad de la República, Uruguay <vicevia@gmail.com>, ORCID: 0000-0001-9049-246
} 


\section{Introducción}

$\mathrm{P}$ arir y nacer es un hecho humano universal, pero, como todo proceso biológico, la manera en que nacemos y parimos está modelada por el entorno social y por sus relaciones. Para la biomedicina, parir es un acto riesgoso $\mathrm{y}$, por tanto, debe atenderse; esta idea de riesgo opera asimismo en las subjetividades de las personas involucradas en un parto y nacimiento. A las inseguridades habituales durante el acto del nacimiento hoy se añaden incertidumbres derivadas de la pandemia por Covid-19 (O’Connell et al., 2020), que llegó a los países de América Latina entre los meses de febrero y marzo de 2020 (Benítez et al. 2020).

Durante el primer año de desarrollo de la pandemia no existían evidencias de que el SARS-CoV-2 fuera particularmente nocivo para las mujeres durante el embarazo, ${ }^{1}$ pero sí hubo reportes de incremento de la prematurez y de restricción de crecimiento fetal (Gandioli, 2021; Briozzo et al., 2020; ops, 2020; Aguirre et al., 2020) que podrían explicarse por los cambios que la pandemia genera en los sistemas de salud y cómo éstos repercuten en la disminución de los controles de embarazos, lo cual aumenta la patología materna perinatal no diagnosticada, afecta la calidad y humanización de los cuidados e incrementa el intervencionismo innecesario (Briozzo et al., 2020). Es decir que, si bien no se tienen reportes concluyentes sobre la afectación específica de la coviD-19 en la salud de la embarazada y el feto en términos biomédicos, las transformaciones sociosanitarias más generales por consecuencia de la pandemia terminan por influir de diversas maneras en este proceso.

Estudios sociales sobre embarazo y parto en la pandemia de COVID-19 señalan que los mensajes de alarma, los cambiantes protocolos sanitarios y las formas erráticas de comunicación de las instituciones sanitarias hacia las mujeres, la saturación de los servicios de salud, entre otros factores, contribuyen a generar incertidumbres y temores respecto de las condiciones de atención al nacimiento en el contexto de la pandemia, y han incidido en las trayectorias de búsqueda de atención (Vázquez-Xu, 2020; Costa Abós y Behaghel, 2020; O'Connell et al., 2020; Souto, Sartori de Alburquerque y Prata, 2020; Santana, 2020;
Castrillo, 2020). A todo esto se suma la percepción de los hospitales como sitios de potencial contagio (Davis-Floyd, Gutschow y Schwartz, 2020; Costa Abós y Behaghel, 2020) y en países como Estados Unidos, España, Portugal, Brasil y Argentina se ha registrado un retroceso en la posibilidad de cumplir con el derecho al acompañamiento durante el parto, con el argumento de prevenir el contagio (Davis-Floyd, Gutschow y Schwartz, 2020; Costa Abós y Behaghel 2020; Souto, Sartori de Alberque y Prata, 2020, Castrillo, 2020). En países como Brasil y Portugal también se ha suspendido la presencia de doulas y visitantes, se ha recurrido a intervenciones obstétricas innecesarias y se han revisado los criterios de contacto piel con piel entre la madre y el o la recién nacido/a, y el amamantamiento inmediato, entre otras prácticas recomendadas por la Organización Mundial de la Salud (oms), lo que podría indicar un retroceso en el respeto de los derechos reproductivos; desde la antropología feminista se ha advertido que en tiempos de crisis y excepcionalidad suele darse un ajuste del cinturón patriarcal (Klein, cit. en Petruccelli y Mare, 2020: 159).

En Uruguay, las recomendaciones del Ministerio de Salud Pública (MSP) para la atención sanitaria al nacimiento durante la pandemia se basan en pautas de la oms, guías clínicas nacionales y abogan por el respeto a la Ley 17.386 (Ley de acompañamiento a la mujer en el preparto, parto y nacimiento, de 2001). ${ }^{2}$ No obstante, organizaciones de la sociedad civil, representantes de organizaciones profesionales ginecoobstétricas y la Institución Nacional de Derechos Humanos (INDDHH) han denunciado que durante la pandemia por COVID-19 se han incumplido derechos reproductivos consagrados en la legislación nacional (INDDHH, 2020; La Diaria, 2020, Arteta, 2020, Briozzo et al., 2020).

Más allá de estas aproximaciones, hasta el momento no se han realizado investigaciones que den cuenta de la experiencia de las protagonistas ¿Cuáles están siendo sus temores e incertidumbres frente al parto en contexto de pandemia? ¿Cómo experimentan el encuentro con los servicios y profesionales de la salud durante la gestación, parto y puerperio? ¿Confían en que sus derechos sean respetados? ¿Cómo actúan frente a la falta de certezas, miedos y posibles

\footnotetext{
1 La evolución de esta enfermedad y sus impactos así como la evidencia biomédica disponible es muy dinámica. Al momento en que fue escrito el artículo y analizados los datos (segundo semestre de 2020) ésos eran los criterios manejados. En el mes de mayo de 2021, cuando este artículo estaba en la última fase de maquetado, comenzó a detectarse un aumento de los casos de embarazadas en estado grave debido a covid-19 en Uruguay; al 8 de mayo dos embarazadas habían fallecido por este motivo. La explicación podría estar en la presencia de la cepa brasileña en el país, pero todavía no está confirmado que éste sea el motivo. El cambio en la situación ha llevado a considerar a las embarazadas como un grupo de riesgo y a priorizarlas en la agenda de vacunación (véase por ejemplo Keusseian 2021).

2 https://www.impo.com.uy/bases/leyes/17386-2001
} 
incumplimientos de las normativas vigentes? ¿Hay evidencias para hablar de un recrudecimiento de la violencia obstétrica en las actuales circunstancias?

En este artículo proponemos acercarnos al parto y al nacimiento desde la experiencia de algunas mujeres que tuvieron sus hijas / os en el contexto de pandemia por Covid-19 en Uruguay, a partir de los aportes de la antropología médica y la antropología feminista. Esperamos visibilizar las relaciones entre las dimensiones estructurales de la producción social del parto y el nacimiento -incluyendo instancias previas y posteriores, como controles obstétricos, controles neonatales y puerperio-, las vivencias de las protagonistas y el modo en el que la sociedad y el gobierno uruguayos enfrentaron la llegada de la covid-19 al país.

\section{Aproximaciones teóricas y situación de Uruguay en el contexto de la región}

Como plantea desde hace décadas la antropología médica, los procesos de salud-enfermedad-atención no son sólo fenómenos fisiológicos, sino que están atravesados y son constituidos por relaciones sociales y culturales y son estructurales en la reproducción biosocial de las sociedades humanas (Menéndez, 2009). Esto incluye tanto a la población como a los diversos sistemas terapéuticos y de atención a la salud. La población posee un conjunto de saberes sobre los padecimientos, así como de las nociones de vulnerabilidad, riesgo y prevención, que se dan en primer lugar en el plano de la unidad doméstica y las redes de apoyo y autoayuda primarias (Menéndez, 2009; Osorio, 2011; Menéndez, 2020).

Como sintetizan Manderson y Levine (2020), desde la antropología médica se ha contribuido a la comprensión de las pandemias y epidemias y sus efectos en la vida social, económica y en los sistemas de atención, desde el estudio de los imaginarios colectivos sobre las plagas hasta el análisis de la expansión e impactos de enfermedades infectocontagiosas como el virus de inmunodeficiencia humana ( $\mathrm{VIH})$, la varicela, el cólera, la hepatitis, el síndrome respiratorio agudo grave (sARs), la influenza H1N1 (gripe porcina), la influenza H5N1 (gripe aviar), el ébola, el zika, entre otras. Estos estudios han demostrado que la cultura atraviesa diversas prácticas y comportamientos clave en eventos de transmisión comunitaria, como la institución de cuarentenas y controles fronterizos, las racionalidades presentes en los servicios de atención sanitaria y hasta las prácticas cotidianas de higiene, aprovisionamiento de alimentos y cuidados en los hogares (Manderson y Levine, 2020).
En especial la reproducción humana, la salud materna y los saberes maternos han sido temas centrales para la antropología médica crítica latinoamericana (Freyermuth, 2003; Osorio, 201 1; Gamlin et al., 2020) y su abordaje ha incluido las preocupaciones sobre derechos reproductivos y desigualdad de género (Gamlin et al., 2020). Se trata de procesos socioculturales atravesados por múltiples formas de comprender la maternidad-paternidad, la salud y la atención, según órdenes culturales y de género y abarcan múltiples ámbitos de la vida cotidiana (Berrio, 2017). El embarazo-parto y puerperio tienen gran importancia, no sólo en el proceso vital de cada mujer, sino también en las relaciones sociales, pues el nacimiento es el momento en que la sociedad recibe a un nuevo ser.

Este interés se articula con el abordaje de la reproducción desde una perspectiva feminista, que ha buscado echar luz sobre la incidencia del género en los modelos de atención y en la experiencia subjetiva de los procesos de embarazo, parto y puerperio. El género es un sistema de organización social de la diferencia sexual, entendida ésta no como una condición anatómica, sino como producto de un trabajo de diferenciación social (Butler, 2005) A partir de estos procesos de diferenciación, el género establece representaciones de lo masculino y lo femenino que penetran en el accionar de las instituciones, e inciden en las subjetividades. Estas diferenciaciones conllevan además relaciones de asimetría y desigualdad, por lo que hablar de género es hacer referencia a un sistema de poder (Scott, 1996), en el que las diferenciaciones sexo-generizadas se intersectan (Crenshaw, 2002) con otras, como la clase social, la pertenencia étnica, la identidad sexual.

En lo que atañe a la fecundación, embarazo y parto, el género está involucrado en las maneras de simbolizar estos procesos (Martin, 1991 y 2001), junto con los modos de comprender la producción social del cuerpo, incorporando la experiencia corporal reflexiva para complejizar "las relaciones entre cuerpos, ideologías, relaciones sociales e instituciones" (Esteban, 2006: 15). La atención al parto y nacimiento en las sociedades occidentales ha estado marcada por un proceso de creciente medicalización (Illich, 1976; Zorzanelli, Ortega y Bezerra, 2014; Davis-Floyd, 2001), que ha puesto en un primer lugar el saber técnico científico de la biomedicina. Este proceso se ha desarrollado en un entorno económico capitalista y en sociedades patriarcales, todo lo cual se conjuga en lo que Davis-Floyd (2001) define como "modelo tecnocrático del parto y el nacimiento", que se ciñe a tiempos institucionales, emulando el modo de producción fabril; sobrevalora la ciencia y la tecnología, prioriza resultados a corto plazo 
y se centra en el objetivo de lograr un "producto" (bebé) sano. Estas concepciones y consecuentes prácticas, emergentes de relaciones estructurales patriarcales que operan en el control de la reproducción como parte del control de la sexualidad femenina (Rodrigáñez, 2009; Gainza, Labastie y Magnone Alemán, 2013; Bellón Sánchez, 2015) y de relaciones capitalistas, que configuran el propio proceso de medicalización del parto y el nacimiento, sostienen la posibilidad de existencia de lo que se ha denominado violencia obstétrica. Ésta alude a la realización de procedimientos obstétricos no debidamente justificados, que pueden ser lesivos para la persona gestante. El concepto y la práctica política de los derechos reproductivos, y particularmente de los derechos relativos a los procesos obstétricos, son una herramienta conceptual y política (Correa y Petchesky, 1996) para reivindicar otras formas de atención, respetuosas de la persona gestante, del bebé y de su entorno, pero también para cuestionar de forma más amplia las relaciones de género imperantes y sus expresiones en prácticas en el campo de la reproducción.

En Uruguay, el MSP promueve el parto humanizado institucionalizado, pero no está prohibido el parto en casa. El proceso de medicalización en Uruguay comenzó en 1900 (Barrán, 1993 y 1999), y con él se institucionalizó la atención al parto y al nacimiento y de manera progresiva se estableció un modelo de atención en consonancia con el modelo tecnocrático descrito por Davis-Floyd (2001), el cual hoy coexiste en Uruguay con un modelo incipiente de parto humanizado (Magnone, 2011). La Ley 18.426 de Defensa del Derecho a la Salud Sexual y Reproductiva, aprobada en $2008^{3}$ busca justamente "promover el parto humanizado garantizando la intimidad y privacidad; respetando el tiempo biológico y psicológico y las pautas culturales de la protagonista y evitando prácticas invasivas o suministro de medicación que no estén justificados" (artículo $3^{\circ}$, inciso 3 ).

El país tiene además otras leyes orientadas a hacer efectivos los derechos obstétricos; la ya mencionada Ley 17.386, que dispone que durante el trabajo de parto y el parto toda mujer tiene derecho a estar acompañada de una persona de su confianza; y la Ley No 17.565 (de 2002), ${ }^{4}$ que ordena que en todo establecimiento donde se asisten partos haya una partera interna de guardia. Desde 2017 se cuenta con la ley de violen- cia basada en género (Ley 19.580), ${ }^{5}$ la cual reconoce la violencia obstétrica como una forma de violencia de género. En la práctica, sin embargo, siguen produciéndose intervenciones que atentan contra estas normativas, como un porcentaje de cesáreas que supera el 10-15 por ciento establecido por la oms -43 por ciento en 2017-, y de episiotomías, práctica realizada en más de la mitad de los partos (Marijanovich, 2019). Al mismo tiempo, según datos de la Organización Panamericana de la Salud (ops, 2010), Uruguay tiene la tasa de fallecimientos, vinculados básicamente al embarazo, parto, puerperio y aborto, más baja de América Latina y el Caribe. Esto podría responder a factores como la exoneración de erogaciones para realizar controles clínicos y paraclínicos del embarazo (Decreto 562/005) ${ }^{6}$ y, desde 2001, antes de la aprobación de la Ley de Interrupción Voluntaria del Embarazo, al desarrollo de diversas medidas para disminuir la muerte gestante por aborto provocado en condiciones de riesgo (Briozzo, 2002).

En cuanto a la pandemia, los primeros casos de Covid- 19 fueron confirmados el 13 de marzo, pocos días después de la asunción de un nuevo presidente, Luis Lacalle Pou (liberal), representante del Partido Nacional. La política del gobierno fue exhortar, sin obligar, al distanciamiento social. Se cerraron los ámbitos donde pudiera haber aglomeración de público, incluyendo los centros educativos, y se instauró el teletrabajo y la educación a distancia; algunas de estas medidas fueron flexibilizándose de manera paulatina. Hasta noviembre de 2020 , cuando comenzó un crecimiento exponencial de los casos, Uruguay había sido reconocido internacionalmente por su baja tasa de incidencia de la enfermedad y las acciones adoptadas para afrontar la pandemia (BBC Redacción, 2020). $\mathrm{El}$ informe epidemiológico de diciembre 2020 reporta 6731 casos acumulados de covid- 19 en Uruguay $(71.70$ por ciento se consideran recuperados); 54 corresponden a mujeres embarazadas, de las cuales 20 estaban activas a la fecha del informe (MSP, 2020). ${ }^{7}$

Respecto de las medidas sanitarias adoptadas para la atención de los partos en este contexto, a mediados de abril de 2020 el MsP publicó las "Recomendaciones para la mujer embarazada y el recién nacido frente a la covid-19" (Aguirre et al., 2020), donde se recomienda la atención del nacimiento con base en las prácticas habituales para las mujeres asintomáticas,

\footnotetext{
3 https: / /oig.cepal.org/sites / default/files /2008_ley 18.426_uruguay.pdf

4 https://legislativo.parlamento.gub.uy/temporales/leytemp965258.htm

5 https: / /www.impo.com.uy/bases / leyes / 19580-2017

https: / / www.impo.com.uy/bases/decretos/562-2005

Esta es la información epidemiológica más actualizada disponible al momento de la elaboración de este artículo.
} 
pero se plantea evaluar el riesgo del acompañamiento en las pacientes positivas (Aguirre et al., 2020: 15). Sin embargo, organizaciones de la sociedad civil denunciaron ante la INDDHH que se estaba vulnerando el derecho al parto humanizado y a la salud familiar integral, pues en centros asistenciales públicos y privados de la capital (Montevideo) y del interior del país se había impedido la presencia de acompañante durante el parto, invocando medidas especiales debido a la emergencia sanitaria (INDDHH, 2020). Fue en este ambiente de tensiones políticas y protocolos sanitarios cambiantes, en función de las variaciones en las condiciones sanitarias del país, que tuvieron lugar las vivencias y la atención del embarazo, parto y puerperio de las mujeres con quienes conversamos.

\section{Metodología}

La metodología utilizada para este estudio es de carácter cualitativo y exploratorio. Combina la sistematización de diversas fuentes (informes epidemiológicos con relación a la covid-19 y su impacto en la salud y en atención sanitaria de gestantes y recién nacidos en Uruguay y la región, documentos oficiales del MsP con recomendaciones para la atención de mujeres embarazadas durante la pandemia en Uruguay y prensa nacional) con la realización de entrevistas abiertas a mujeres que parieron durante el primer semestre del transcurso de la pandemia.

Se entrevistó a ocho mujeres, en una franja etárea que va de los 20 a los 40 años. En las entrevistas se resaltó la experiencia del nacimiento en el contexto de emergencia sanitaria, así como aspectos relativos a los controles prenatales en pandemia, los cuidados de la mujer durante el puerperio y los primeros cuidados del/la recién nacido/a.

El tiempo transcurrido entre el parto y la realización de la entrevista fue diverso, lo cual incidió en el ejercicio de reflexividad de cada una de las mujeres acerca de su experiencia. Por otro lado, estas diferencias nos permitieron sopesar en mejor medida el factor de la temporalidad en la gestión sanitaria de la pandemia y su incidencia en el modo de atención del proceso de embarazo y parto y, por tanto, también en la construcción de la vivencia de este proceso.

Por tratarse de un estudio exploratorio, se dio prioridad a la diversidad de experiencias de nacimiento y tipo de parto (vaginal y cesárea), así como la multiplicidad de prestadores de salud. Todos los nacimientos considerados sucedieron en Montevideo, y todas las entrevistadas viven en Montevideo o área metropolitana-localidades cercanas a la capital.
Entrevistamos a mujeres que reciben asistencia de los principales tipos de prestadores de salud en Uruguay, tanto de la Administración de Servicios de Salud del Estado (ASSE), de instituciones de asistencia médica colectiva (IAMC) y de seguros privados. Debido a cómo fueron captadas las participantes del estudio trabajamos sobre todo con usuarias de IAMC y seguros privados. Estrategias desplegadas en estudios previos de las autoras (Mesa y Viera Cherro, 2004; Evia, 2016), como el contacto cara a cara en salas de espera de centros asistenciales o referencias de personal de salud, no fueron viables por las limitaciones para el acceso; recurrimos entonces como vía primordial para contactar a las entrevistadas a nuestras redes de amistad, familiares y laborales, lo cual nos condujo a un grupo de entrevistadas con un perfil socioeconómico relativamente homogéneo, con predominio de mujeres con educación terciaria e inserción laboral profesional, condición que, por una parte, impide generalizar las conclusiones hacia otros estratos sociales, pero que al mismo tiempo permite profundizar, desde un punto de vista analítico, en lo que se considera particular de este grupo social.

En la figura 1 se sitúan en orden cronológico según la fecha de los partos y tipo de prestador de salud las principales características de las entrevistadas en relación con los principales hitos de las políticas de gestión de la pandemia vinculadas al parto.

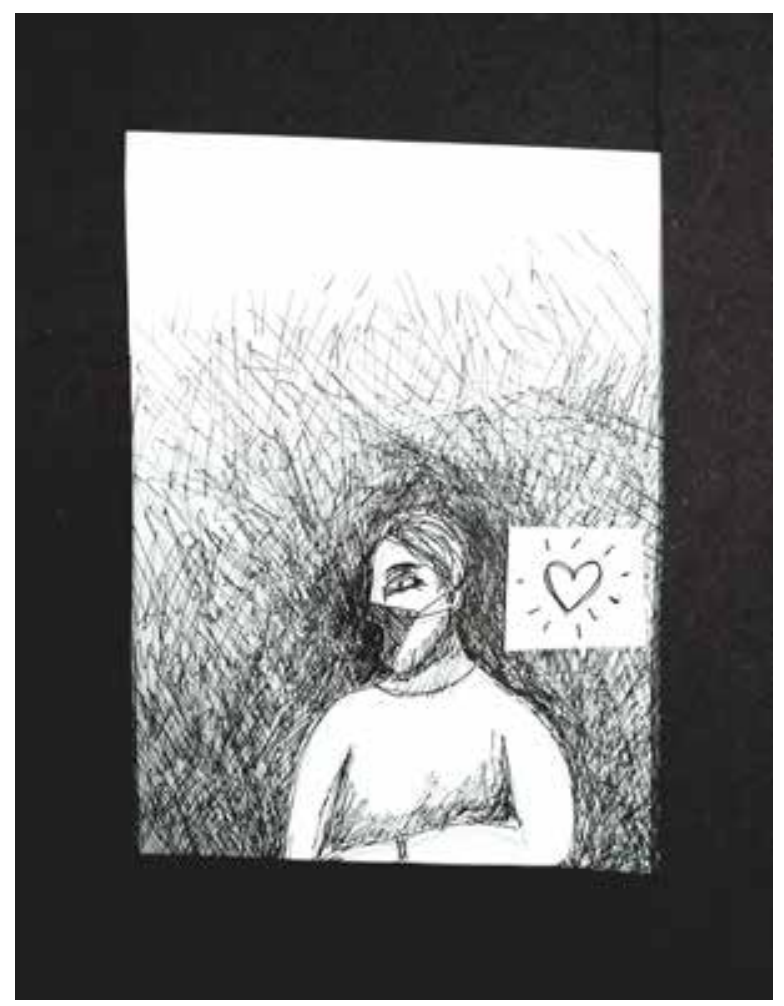




\section{Figura 1}

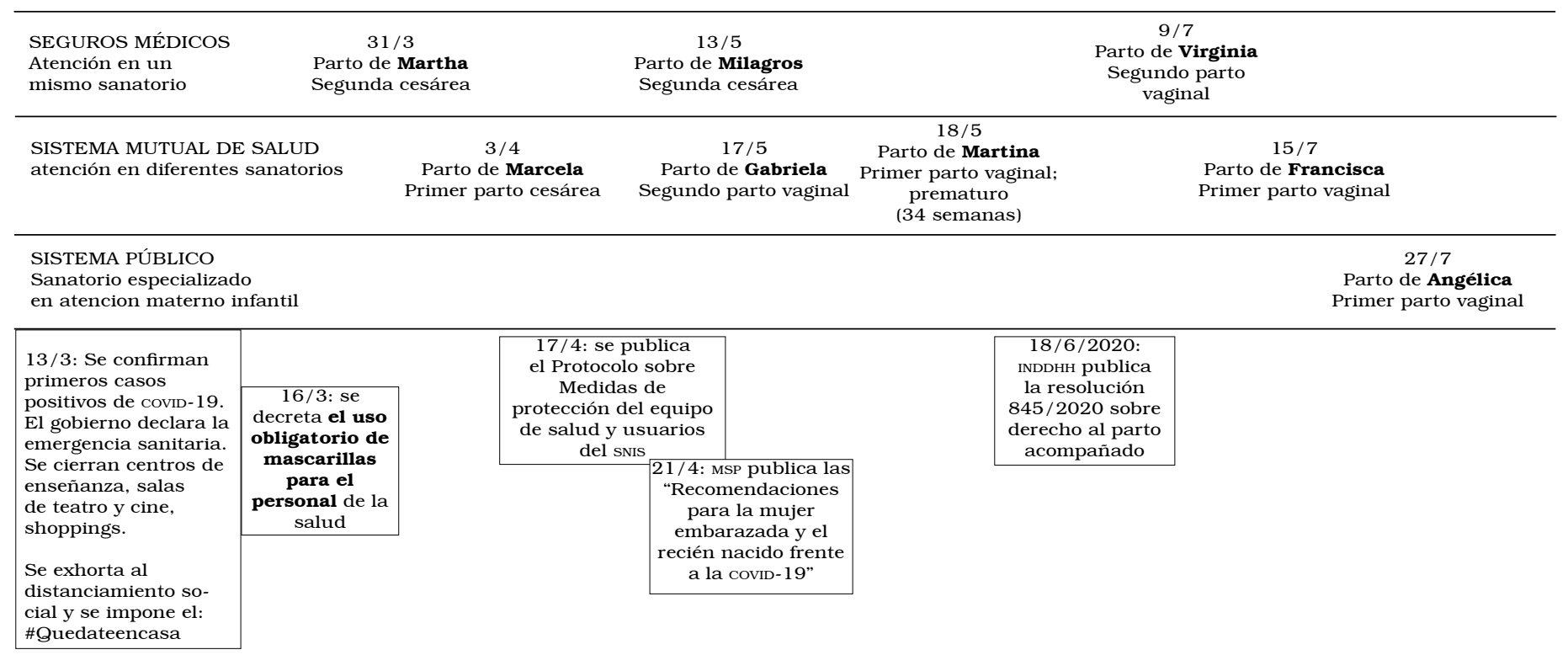

Las entrevistas se llevaron a cabo sobre todo por teléfono o videollamada, excepto una que, debido a la preferencia de la entrevistada, se realizó de manera presencial fuera de su hogar. A pesar de que en Uruguay el confinamiento no fue obligatorio, quienes pudieron se plegaron al "\#Quedarse en casa”. Adicionalmente, la sobrecarga de cuidados domésticos debido a la suspensión de clases presenciales en enseñanza primaria y secundaria hizo que quienes tenían hijas/os a cargo (entrevistadas y entrevistadoras), dispusieran de menos tiempo para encuentros presenciales. Como señalan Aparicio et al. (2020) en un estudio sobre estrategias de cuidado en la pandemia entre mujeres del conurbano bonaerense, las entrevistas mediante aplicaciones de videollamada y mensajería por redes sociales son útiles para facilitar el diálogo con mujeres a quienes el sostenimiento del hogar y las tareas de cuidado les exigen mucho.

Efectuar las entrevistas por videollamada posibilita contemplar las reacciones de la otra persona, habilita la repregunta y en cierto sentido emula el encuentro cara a cara; también permite ingresar a la intimidad de una persona en situación de puerperio -como lo estaban la mayoría de las entrevistadas- de forma menos intrusiva en relación con ingresar presencialmente al hogar. Las aplicaciones de mensajería (de texto o audio) allanaron la coordinación de los encuentros e incluso la repregunta en un diálogo que podía ser simultáneo o asincrónico, según los tiempos disponibles. No obstante, reconocemos que este tipo de herramienta puede presentar limitaciones, pues intermedia en la interacción entre entrevistadora y entrevistada, lo cual genera un efecto de distanciamiento que impacta las dinámicas de transferencia y contratransferencia implicadas en cualquier entrevista (Robben, 2011). A pesar de lo anterior, creemos que el uso creativo y reflexivo de las tecnologías de la información y la comunicación hicieron factible la realización de este estudio, aun en las presentes circunstancias.

En todas las entrevistas se obtuvo el consentimiento oral de las participantes y se les aseguró anonimato y confidencialidad en el manejo de la información. Los nombres en el artículo han sido modificados con estos fines.

\section{Incertidumbres, miedos, estrategias y temporalidades pandémicas}

De los relatos de las entrevistadas queda en evidencia el peso de la temporalidad del desarrollo de la pandemia, tanto en sus temores e incertidumbres durante el embarazo como en los cambios en los encuentros con los servicios de salud en los distintos momentos de la atención (controles prenatales, postnatales y parto) (véase figura 1).

Los cambios en las medidas de atención, al compás de la información sobre el impacto que podía tener la enfermedad en las gestantes y en los/as recién nacidos/as, de lo que en un principio se sabía muy poco, llevaba a incertidumbres diversas y en función de estas diferentes etapas. 


\section{El embarazo y "no saber qué esperar"}

Para las mujeres que cursaban su embarazo en el mes de marzo, la declaración de la emergencia sanitaria implicó un cambio en su vida cotidiana junto con el resto de la sociedad, así como modificaciones en la atención sanitaria y preocupaciones respecto de la potencial peligrosidad de esta enfermedad "desconocida" en esta etapa vital, tanto para la mujer como para el feto. El aislamiento social voluntario, la instauración del teletrabajo desde la unidad doméstica, la suspensión de actividades escolares en los casos de familias con más hijos, el desasosiego por el estado de salud de parientes mayores y en general por el deterioro de la situación económica en el país, entre otros factores, agregaron estrés a esta etapa vital y una mayor carga de trabajo doméstico en tareas de higiene y cuidado.

En cuanto a los potenciales riesgos biológicos que la Covid-19 tuviera para el embarazo y el bebé, las circunstancias fueron variando de acuerdo con la evolución de la temporalidad de la pandemia, al momento del nacimiento y a la información disponible para las mujeres.

Martha, que tuvo a su hija el 31 de marzo: "no sabía qué esperar”. La cesárea de su hija, la cual tuvo que programarse a las 37 semanas porque Martha estaba con presión, al ser tan cercana a la llegada de la COVID-19 a Uruguay, supuso una mayor incertidumbre. Su misma doctora le había advertido que las formas en que se estaba llevando la atención cambiaba "día a día” "estaban probando y me generó miedo, porque yo no sabía. Por más que me decían que el bebé no se iba a contagiar, te da miedo" (entrevista, 7 de septiembre).

Por su parte, Gabriela, que parió en mayo, afirmaba no tener miedo de la CoviD-19 respecto de sí misma, porque "sabía" que la enfermedad no conllevaba un riesgo especial en su condición. No obstante, le preocupaba que en caso de enfermedad o muerte de sus familiares no pudiera ayudarlos por estar embarazada y entrar socialmente en una categoría de persona "vulnerable". Por último, Francisca, buscó informarse acerca de estos riesgos, "Participé de una charla online gratuita de una española que hacía un racconto de todos los estudios en embarazadas y los riesgos para el recién nacido y ahí me fui tranquilizando, porque los estudios decían que no había gran riesgo [...]. Me dejó tranquila saber que el virus no pasaba por la placenta" (entrevista, 18 de septiembre).

Al referirse a la atención sanitaria, Martha señalaba que al comienzo hubo cambios en los criterios habituales. Todas las entrevistadas mencionaron cancelaciones o reprogramación de las consultas y rutinas de control del embarazo, así como mensajes disuasorios más o menos explícitos de asistir a, y/o permanecer en, los servicios de salud (mensajes de texto, cambios en la disposición del espacio-barreras físicas, señalética-, de rutinas para el ingreso -revisión de síntomas respiratorios, sanitización de manos y control de temperatura-), además de no poder ir acompañada a los controles ginecoobstétricos y a las ecografías. En algunas excepciones, la posibilidad de acompañamiento pudo ser negociada con el médico tratante, sin embargo, cada encuentro con el sistema de salud era experimentado con cierta incertidumbre por los potenciales cambios en el "protocolo".

Marcela, que tuvo a su primer hijo a término el 3 de abril en una IAMC, al poco tiempo de instalada la emergencia sanitaria, nos decía, "Fue como rara la época que me agarra a mí; todavía lo del tapabocas no estaba del todo establecido [...] [era el] momento que el tapabocas no era obligatorio, incluso algunos decían que no era bueno tenerlo". Asimismo, relata cambios en el funcionamiento de la mutualista (fundamentalmente recortes en las horas de dedicación del personal médico) que afectaron la atención de su embarazo:

la última semana antes de tener, tenía ginecólogo, voy y ahí me avisan que el horario a los ginecólogos se les había reducido y a mí nadie me avisó nada, yo estoy a una semana de parir, yo ahí me quedé enojada con la mutualista [...] lograron que me atienda otro ginecólogo, que no era el mío [...] por lo menos me quedé tranquila de que alguien me había visto (entrevista, 2 de septiembre).

A Gabriela, le reprogramaron los controles de seguimiento del embarazo y el principal cambio para ella fue que ya no pudo ir acompañada; tampoco a algunas de las ecografías obstétricas. Para la primera ecografía en pandemia ni siquiera consideró que fuera una posibilidad. "Ir sola a los controles te puede joder la atención. Puede cambiarte la vivencia de esa etapa" (entrevista, 17 de septiembre). Además, ella buscaba informarse sobre qué otros cambios en la atención podían derivarse del contexto de pandemia: "Yo desde que empezó la pandemia preguntaba si había alguna novedad sobre los protocolos y la respuesta era siempre 'ninguna'. Si tenés síntomas de infección respiratoria (tú o tu pareja) se van a activar los 'protocolos covid'[...] Pero no se sabía mucho tampoco qué era eso. Quedaba muy 'técnico dependiente'." Esto denota el temor por la posibilidad de la arbitrariedad en la implementación de los protocolos según los criterios, no sólo de cada institución, sino incluso del técnico de turno, y la aceptación tácita de que, desde el lugar del paciente, no habría mucho que se pudiera hacer. 
Si bien en la guía del MSP (Aguirre et al., 2020) se recomendaba la posibilidad del acompañamiento en controles obstétricos y ecografías en caso de mujeres no asintomáticas, la experiencia de la mayoría de las entrevistadas durante los primeros meses coincidió con la de Gabriela y tuvieron que ir solas a estos estudios. Angélica, que tuvo a su primer hijo el 27 de julio en un centro asistencial público, grababa las ecografías para mostrárselas luego al papá de su hijo, que aguardaba afuera del consultorio. Algunas pudieron negociar el acompañamiento en el marco de una atención médica por seguro privado, y otras cuando ya las condiciones de atención parecían ser más flexibles. Milagros, cuyo bebé nació el 17 de mayo, con atención del seguro privado, asumió que tenía que ir sola a las ecografías, pero pudo estar con su compañero durante las consultas de control del embarazo.

En el caso de Gabriela (sistema mutual), sí pudo ir con alguien a la ecografía del tercer trimestre a comienzos de mayo, aunque no lo había hecho en ecografías anteriores. Estos cambios pueden haber obedecido a la mayor laxitud en las condiciones de atención pasado el mes de abril cuando la incidencia de la enfermedad parecía controlada, así como a los efectos de las denuncias presentadas por organizaciones de la sociedad civil y las recomendaciones realizadas por la INDDHH al MSP de velar por el respeto al derecho al acompañamiento (INDDHH, 2020).

Francisca - que pudo negociar con su médica tratante el acompañamiento en los controles obstétricoscuenta que, como en las ecografías la decisión de entrar o no acompañada "quedaba librada al técnico", ella y su pareja decidieron hacerse una de las ecografías de control en una clínica privada para asegurarse de ir juntos. "Cuando pagás, tenés lo que querés", sentencia (entrevista, 18 de septiembre). Para minimizar incertidumbres en el proceso de atención ellos optaron por pagar un estudio privado, cuando por el Sistema Nacional Integrado de Salud (sNIs) tenían derecho a realizarlo sin costo. Esto muestra cómo las condiciones de atención durante la pandemia inciden en las decisiones sobre las trayectorias de atención de las mujeres $\mathrm{y}$ alerta sobre los potenciales incrementos del gasto de bolsillo en salud.

La visualización del embarazo y el parto como instancias de riesgo es constitutivo del modelo tecnocrático de atención al parto y el nacimiento (Davis-Floyd, 2001); es también esta idea de riesgo la que modela la forma subjetiva de transitar este proceso y las expectativas de atención del mismo. Como advierte Silvia Sosa, partera y cofundadora en 2012 de la Organización Civil "Nacer Mejor", en una entrevista radial a fines de abril sobre embarazo y parto en el contexto de la pandemia,

Durante años nos inculcaron que ir regularmente a la consulta nos garantizaba un nacimiento saludable [...] Nos repitieron en muchas campañas de promoción de seguimiento del embarazo [...] en ese proceso las mujeres dejamos de confiar un poquito en nuestros cuerpos y pasamos a depender de la mirada del médico y de las ecografías.

Desde este marco podemos comprender la incertidumbre y el temor con el que vivieron algunas de las mujeres entrevistadas la cancelación de consultas de seguimiento del embarazo. No hablamos de desestimar la envergadura de estas consultas ni desconsiderar que hay aspectos clínicos del embarazo que es esencial evaluar y cuyo control no puede hacerse a través de una videollamada, pero es importante sopesar esta incertidumbre en diálogo con las expectativas de atención que naturalizan en nuestro país la necesidad de realizar de diez o doce controles médicos durante el embarazo. Al mismo tiempo, en el contexto de la pandemia las recomendaciones de espaciar estos controles, sugiriendo que se efectúen de cinco a seis controles durante el embarazo normal (Briozzo et al., 2020), invita a relativizar la necesidad de estos controles. ${ }^{8}$

En cuanto a la vida cotidiana durante el embarazo, las propias entrevistadas identifican diferentes fases temporales en la vivencia de la pandemia y cómo fueron variando sus prácticas preventivas. Si bien se refirieron a prácticas preventivo-sanitarias (higiene personal y doméstica, uso de tapabocas, entre otros) el principal énfasis estuvo puesto en las medidas de distanciamiento social o cuarentena, fomentadas por el llamado que, desde las políticas públicas y desde la sociedad toda, se hacía al \#Quedateencasa.

Francisca y su compañero tomaron medidas de aislamiento voluntario para disminuir las posibilidades de contagio del virus. Ella periodiza esta etapa entre marzo y mediados de junio, y reflexiona sobre las circunstancias que enmarcaban esta decisión: la presencia del coronavirus de forma cotidiana y esta catalogación social de las embarazadas como un grupo de riesgo. Luego, un mes antes del nacimiento del bebé, en junio, "habilitamos algunos contactos. Porque si no, no me agarraba el coronavirus, pero me

\footnotetext{
8 "Embarazo y nacimiento en tiempo de coronavirus", entrevista realizada por Andrea Cammarano el 24 de abril de 2020 , disponible en: https: / /youtu.be/X3F8eqJFHhk [31 de octubre de 2020].
} 
agarraba tremenda depresión”. Gabriela también refirió haber adoptado medidas de cuarentena voluntaria muy estrictas con su familia hasta el nacimiento de su hija, como forma de prevenir el contagio de algún miembro del grupo familiar, no tanto por temor a la COVID "en sí", sino por el miedo de que ante cualquier síntoma respiratorio se vieran sometidos a algún criterio técnico que la hiciera "tener que parir sola". Sin embargo, luego del nacimiento empezaron a abrir los contactos sociales e incrementar las salidas al aire libre, considerando que lo que "perdían" en calidad de vida con el encierro suponía un mal mayor que un potencial contagio.

Las prácticas de prevención orientadas a regular cuándo abrir y cuándo cerrar los contactos sociales con otras personas cercanas operan en función de la valoración de los diferentes riesgos y vulnerabilidades que se ponían en juego en cada momento (Osorio, 2011). Por ejemplo, mientras que en una primera etapa ambas optaron por limitar los contactos interpersonales como forma de prevenir el contagio de COVID-19, en una etapa posterior privilegiaron la prevención de la angustia o depresión por el aislamiento y la soledad.

\section{Parir sola, parir acompañada}

Las principales incertidumbres y temores respecto del parto estaban centrados no en la enfermedad en sí, sino en las consecuencias que la pandemia tendría sobre la atención recibida. Algunas preocupaciones compartidas eran de índole "práctico", en especial si se iba o no a solicitar el uso del tapabocas durante el nacimiento, pero las más sustantivas eran acerca de la posibilidad de que algunos derechos consagrados se hicieran efectivos al momento de parir. El temor más acuciante y compartido fue el de "parir sola".

Este temor se acompañó de las noticias en medios de prensa de algunas situaciones en las que este acompañamiento efectivamente no se permitió, lo cual, como ya comentamos, llevó a varias organizaciones a realizar denuncias a través de la prensa y frente al INDDHH. La denuncia identificaba casos donde se había prohibido el acompañamiento durante una cesárea, pero también fuera de la capital del país se constató la prohibición de ingreso de acompañantes a partos vaginales (Sosa, 2020).

Martha, que tuvo su segunda cesárea el 30 de marzo, no pudo estar con su pareja y padre de la niña; su ginecóloga se disculpó “todos los días lo estamos cambiando, pero hoy es así y no puede subir nadie al block contigo". Cuando lo cuenta Martha enfatiza la pena de que su compañero y papá de la bebé no haya podido participar del nacimiento. Pero la vivencia de Martha reviste otras complejidades porque, cuando su ginecóloga le planteó que iban a tener que realizar otra cesárea, lo único que ella le pidió fue que le dieran anestesia general, porque su experiencia anterior con la anestesia epidural había sido "horrible". Cuando supo que su esposo no iba a poder estar en el quirófano, "estar dormida" ya no era lo mismo; le dio miedo. Esto supuso una reevaluación de la mejor opción de anestesia en esas circunstancias; sopesando sus temores, y luego de discutirlo con la ginecóloga, decidió aceptar la anestesia epidural.

Otras entrevistadas, que tuvieron sus partos/nacimientos luego de fines de abril, pudieron anticiparse a la situación. Francisca, que tuvo a su hija el 15 de julio, habiendo escuchado de la prohibición del acompañamiento, nos contaba que estaba "aterrada de que sucediera algo así. Lo que hice fue plantearlo en las clases de parto en [institución mutual]. Ahí me garantizaron que eso era una ley y no se podía violar por más que estuviéramos en pandemia, más allá de que se podrían tomar cuidados con los acompañantes".

Martina, que tuvo un hijo prematuro el 18 de mayo, nos dijo que no poder estar con alguien al momento del parto era su mayor preocupación:

me entró bastante miedo, porque yo no me imaginaba en ese momento estando sola [...]. Entonces mandé un mail a la gerencia del sanatorio y a la Sociedad Uruguay de Ginecotología y me respondieron inmediatamente de los dos lados, diciendo que por lo menos hasta ese momento no había ningún protocolo que dijera que el parto no fuera acompañado. [...] yo tenía el derecho a estar acompañada durante el parto y eso se tenía que respetar [entrevista, 24 de julio].

Si bien con excepción de Martha todas las entrevistadas pudieron ejercer su derecho de parir acompañadas, nos es dable afirmar que el temor a que ese derecho se viera vulnerado fue verbalizado como un temor omnipresente en los relatos de todas las mujeres, eclipsando, o por lo menos poniendo en un segundo plano, otros miedos y vulneraciones de derechos, como la realización de procedimientos obstétricos no debidamente justificados. Marcela -que tuvo su primer hijo el 3 de abril con asistencia mutual- nos cuenta cómo llegó a la cesárea luego de ingresar con ocho centímetros de dilatación en el sanatorio y ser atendida por el ginecólogo de guardia,

cuando llegué me dijeron en una horita nace, intenté una horita más, y a la hora viene el ginecólogo y me dice vamos a cesárea, así que ahí marché a cesárea. [...] me queda 
la duda de si hubiera intentado un poco más... porque en realidad no había nada en concreto de por qué yo fui a cesárea [...] lo que el médico me manifestó es que ya habían pasado cuatro horas [...] obviamente estaba un poco cansada, sí.

De su relato se desprende que no medió explicación por parte del especialista acerca del por qué de la cirugía. Y a nuestra pregunta sobre si pudo estar acompañada, contestó, "Sí, por suerte sí. Fue lo primero que pregunté, cuando me dijeron que nos íbamos a cesárea [...] conozco una amiga que no pudo y que le afectó bastante, el no haber podido tener esa compañía”.

Adicionalmente, la mayoría de las mujeres desplegaron sus estrategias para preservar el derecho a estar acompañadas durante el parto: intercambio de información entre redes de embarazadas, organizaciones sociales, doulas y parteras respecto de los derechos de las mujeres y circulación de información sobre las instituciones que estaban incumpliendo; consultas a sus médicos tratantes sobre el estado de los protocolos; comunicaciones formales con los servicios de salud; así como estrategias de prevención y autoatención en la vida cotidiana para evitar el contagio de la enfermedad previo al nacimiento. Esto es coincidente con los hallazgos de Castrillo (2020) sobre derechos sexuales y cyberactivismo en Argentina.

También importa mencionar que todas las entrevistadas evaluaron el trato del personal de la salud como muy bueno. Martha, que ya había tenido otra experiencia de nacimiento, consideraba que en este contexto "Todos estaban como mucho más atentos al paciente" (entrevista, 7 de septiembre). También Virginia, cuya anterior experiencia de parto había sido en el extranjero evaluaba "Acá lo que es mucho mejor es todo el servicio de enfermería” (entrevista, 9 de julio); por su parte, Martina, con la experiencia de un parto prematuro, decía "A pesar de que fue una situación un poco complicada, porque con 34 semanas no era lo que yo me imaginaba ni era el escenario ideal para que naciera el bebé, [... ] me quedé con un recuerdo relindo" (entrevista, 24 de julio), y lo mismo evocaba Angélica (entrevista, 22 de septiembre) de su experiencia en el hospital público.

En cuanto a las medidas sanitarias adoptadas durante el nacimiento y la internación hospitalaria específicamente destinadas a prevenir la COVID-19, las más mencionadas fueron el chequeo verbal de síntomas respiratorios al momento de ser admitidas en el hospital, la sanitización de manos, el uso de tapabocas por parte del personal de salud -por momentos no tan riguroso-, la exigencia de uso de tapabocas al acompañante durante el trabajo de parto y parto y la prohibición de visitas (con excepción del acompañante designado/a) durante la internación. A todas las mujeres que tuvieron parto vaginal se les permitió la libertad de movimiento -exceptuando la circulación en los pasillos- y el no uso de tapabocas durante el trabajo de parto. Si bien ninguna cuestionó la necesidad preventiva de la suspensión de visitas durante la internación hospitalaria, esto fue vivido con cierta ambivalencia por no poder compartir los primeros momentos del recién nacido/a con las redes sociales más cercanas.

\section{Después del hospital}

Luego del nacimiento se inicia el puerperio de la mujer y empiezan las inquietudes por los cuidados del recién nacido, tanto en el ámbito doméstico como en lo que respecta a los controles sanitarios. Si bien las mujeres también requieren de cuidados específicos en esta etapa, la gran mayoría los minimizó en sus relatos.

Cuando el parto/nacimiento es institucionalizado un momento clave es el alta. En las narraciones de las entrevistadas un elemento novedoso, sobre todo para quienes tenían experiencias de partos anteriores, fue lo que vivieron como un alta en cierta medida anticipada a lo que eran sus expectativas y que, si bien evalúan como comprensible en el contexto de la pandemia, también vivieron como algo para lo que muchas no se sentían preparadas. "No terminó el segundo día en el sanatorio y me dijeron andate, porque estaban por llegar los del Greg Mortimer; entendí por qué me tenía que ir, que era todo un riesgo [...] la primera semana fue complicada acá en casa, pasé muy dolorida [...] no estaba preparada para irme" (entrevista, 7 de septiembre).

La pauta del MsP indica que, si todo va bien, el/la bebé recién nacido/a tiene un control en salud entre los siete y diez días de vida y luego a los 30 días. Estas instancias implicaron nuevas negociaciones e incertidumbres en el encuentro con los servicios de salud. Salvo el caso de Martina, cuyo hijo fue prematuro y recibió una atención especial, los controles de los y las demás recién nacidos / as se efectuaron sin complicaciones, pero las condiciones en las cuales se realizaron fueron diversas: en el servicio de salud donde pudo entrar sólo la madre o aquellos donde concurrieron ambos padres, controles donde el equipo de salud acudió al domicilio o consultas telefónicas.

Angélica, que se atiende en la Asse, mencionó la dificultad de ir a los controles neonatales sin su pareja, pero otras familias que se atendían en seguros 
privados y en IAMC pudieron entrar ambos a la consulta pediátrica. Dos excepciones respecto de la atención en IAMC fueron Marcela y Francisca.

Para Marcela, que tuvo familia a principios de abril, el control neonatal del primer mes de vida fue realizado por teléfono. "Al mes me dijeron que era telefónico, y la verdad que no estuve en nada de acuerdo con que fuera telefónico, porque yo, madre primeriza, tenía un montón de consultas, sobre el peso... tuve que pesarlo yo en una balanza, pesarme primero yo y después con él, que mi balanza no sé qué tan segura es”. En el caso de Francisca, en otra IAMC, se fueron de alta sin las consultas agendadas y en el servicio de salud les indicaron que los iban a llamar por teléfono para coordinar la visita domiciliaria. "Volvimos a casa el viernes y se hizo el miércoles y no nos habían llamado. Ella tenía una semana, la primera consulta es entre los siete y los diez días. Llamamos y nos dijeron que el viernes iban a venir, pero ¿qué hubiera pasado si nosotros no llamábamos? La segunda consulta también fue en domicilio. También llamamos nosotros".

Aunque en la mayoría de los casos se trataba de mujeres que tenían parejas consolidadas, con una condición económica estable y acceso a derechos sociales de licencia pagada por maternidad y paternidad -menos en el caso de Angélica, cuyo compañero realiza trabajos por cuenta propia y de forma esporádica-, afrontar los cuidados de un recién nacido y de la mujer durante el puerperio requiere mucho trabajo y ayuda. Esto conlleva un desafío particular en un contexto de emergencia sanitaria, donde la recomendación generalizada es la del distanciamiento físico, lo cual dificulta apelar a las redes de ayuda mutua de amigos y parientes que se suelen activar en esta etapa.

Ante estas dificultades también se observa la importancia de las estrategias desplegadas con originalidad por estas mujeres y sus familias. Por ejemplo Martha, que además de tener a su bebé ya tiene un hijo de siete años, tuvo a su mamá en cuarentena para que luego del nacimiento pudiera ayudar con el otro hijo (entrevista, 7 de septiembre). En la familia de Gabriela, igualmente con otro niño en casa, recurrieron a una estrategia similar.

Por último, exploramos con las entrevistadas las medidas preventivas respecto de la COvID-19 con sus bebés. Un hallazgo de interés fue que esta enfermedad no era particularmente temida por sus efectos en los bebés. Entre las medidas preventivas adoptadas en el grupo doméstico se mencionaba "los recaudos habituales para los cuidados de un recién nacido/a", como la higiene de manos, no exponerlos a personas con infecciones respiratorias, cuidar la ventilación de los ambientes y no recibir muchas visitas.

\section{Reflexiones finales}

Con base en algunas preguntas que orientaron nuestro trabajo-concernientes a los temores e incertidumbres frente al parto en contexto de pandemia en Uruguay-, este artículo se propuso visibilizar la experiencia de las mujeres que tuvieron hijas / os en estas circunstancias y cómo se dieron los encuentros con los servicios y profesionales de salud, el cumplimiento de los derechos reproductivos y las estrategias desarrolladas por las mujeres.

Nuestros resultados indican que, para el grupo estudiado, los principales temores e incertidumbres en torno al parto y nacimiento durante la pandemia de COVID-19 se centraron más en los potenciales cambios de las condiciones de la atención sanitaria y sus consecuencias, que en el temor a la infección en sí misma. El miedo a parir solas resultó ser el más angustiante, pero también resultaron significativas las incertidumbres ocasionadas por las limitaciones para concurrir acompañadas a controles obstétricos durante el embarazo, a la realización de ecografías y a la atención pediátrica del recién nacido/a.

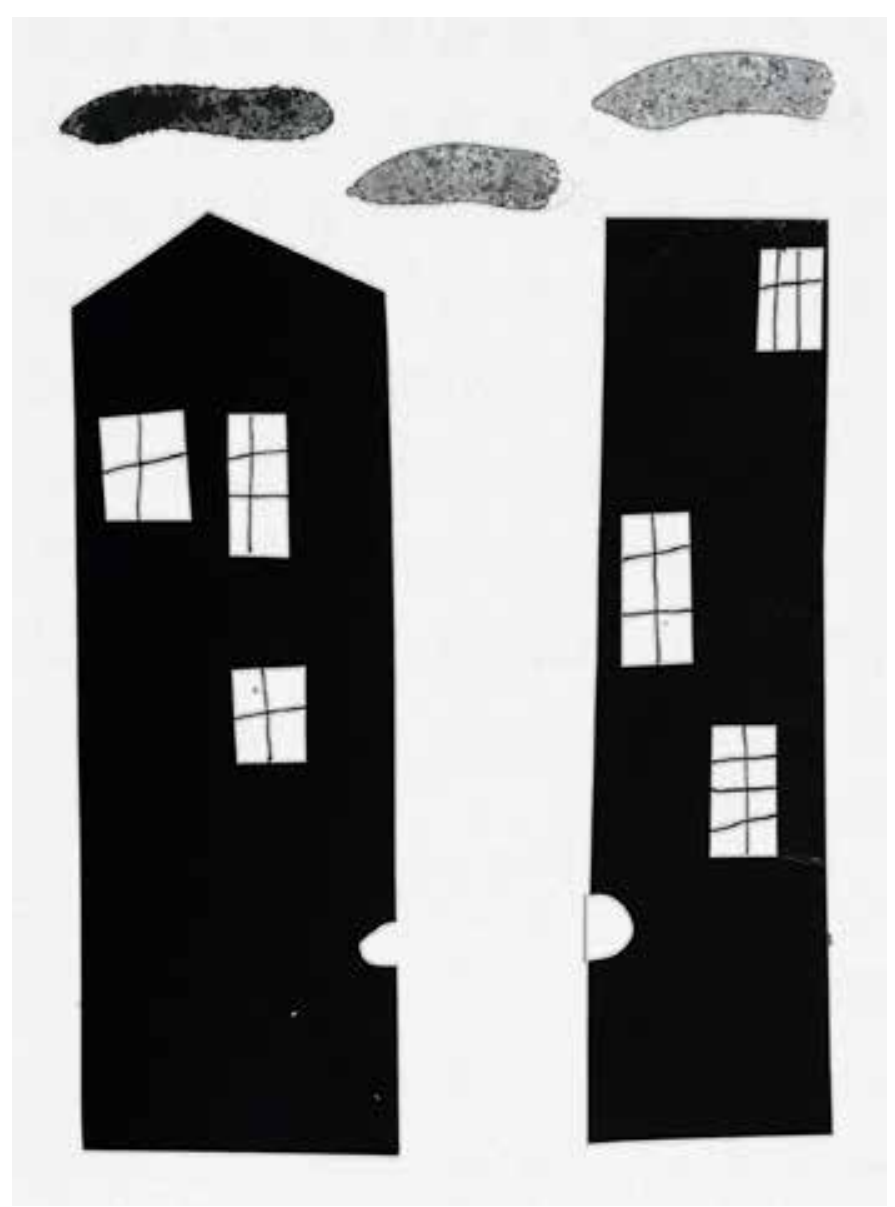


Esto fue así para un grupo de mujeres con nivel educativo alto, que cuenta con inserción laboral profesional, con derecho a la seguridad social, donde la dimensión económica no se vio afectada por la pandemia y en un entorno nacional donde el derecho a la atención sanitaria está ampliamente garantizado. La mayoría de las mujeres entrevistadas, a excepción de Angélica, no "porta" marcas raciales, étnicas o de clase que las coloque en condición de vulnerabilidad específica por estos motivos; Angélica vive en un barrio pobre y no tiene estudios terciarios ni trabajo formal. Como sugieren los antecedentes (Souto, Sartori de Albuquerque y Prata, 2020; Castrillo, 2020; DavisFloyd, Gutschow y Schwartz, 2020; O'Conell et al.; 2020, Santana, 2020), el temor a la vulneración del derecho a parir sola está presente en distintos países y poblaciones, pero otros riesgos percibidos varían según las situaciones nacionales, sus sistemas de salud y las características de clase y racialización de los conjuntos sociales. Por otra parte, si bien los antecedentes indican un aumento de nacimientos en domicilio en países como Estados Unidos y España, como consecuencia de la pandemia, en esta investigación no tuvimos acceso a información significativa al respecto. Todos los casos analizados fueron nacimientos institucionalizados.

Debido a los factores ya analizados, consideramos que los servicios de salud colocaron a estas mujeres en una posición de vulnerabilidad, al obstaculizar el apoyo emocional de un acompañante de su elección en las instancias de atención sanitaria, amplificando los temores e incertidumbres propios de esta etapa vital. Más allá de "excepciones", transacciones o acuerdos entre partes, lo que subyace es la posibilidad de la arbitrariedad en la aplicación de medidas y protocolos que, alegando la excepcionalidad de la coyuntura, vulneran derechos.

Desde un análisis de género, esta apelación al contexto de excepcionalidad para negar el acceso a derechos consagrados implica un recrudecimiento y una profundización de las relaciones de asimetría. Estas relaciones son siempre estructurales, aunque puedan o no evidenciarse en relaciones interpersonales -en las experiencias de las mujeres aparece lo contrario, ya que todas refieren al buen trato asistencial que recibieron-. Estas condiciones patriarcales de sujeción estructural se articulan, en relación con el parto, con un modelo de atención atravesado por lógicas capitalistas. Y, si bien estas lógicas estructurales se evidencian en algunas prácticas recuperadas por las mujeres en sus relatos -como cuando Marcela dice no saber exactamente por qué se le practicó una cesárea-, en este contexto quedan en un segundo plano frente a la mayor preocupación: el parir solas. Las lógicas de género quedan de manifiesto también en la preocupación asociada al hecho de que el varón/ padre no pueda asistir al parto y / o a la realización de estudios -la "pena" que menciona Martha-, poniendo en segundo plano su necesidad de estar acompañada.

Las mujeres recibieron un trato de parte del personal de la salud que juzgaron respetuoso, al tiempo que en sus relatos se revelan diversas formas de violencia, más allá de que fueran o no percibidas como tales por las propias mujeres. No vamos a detenernos aquí en estos eventos, porque trascienden la situación de pandemia y porque no fueron mencionados de manera específica por las entrevistadas. Lo que se vulneró en una ocasión y se puso en entredicho en todas, por lo menos en términos de disposición subjetiva, fue el derecho a estar acompañada al momento de parir. Ello, además de generar temor, muestra la fragilidad política de los derechos reproductivos, fundamentalmente en circunstancias de excepción, el argumento de la excepción se esgrime como razón para no cumplir con dichos derechos. Con todo, como contraparte, la respuesta de la sociedad civil y de algunas instituciones como la INDDHH y el MSP, exhibe al mismo tiempo la politización de estos derechos en la actualidad en Uruguay, así como la importancia de continuar "observando" las actuaciones en el ámbito de la salud. Podemos señalar que un elemento político emergente de lo sucedido con la atención al parto y al nacimiento durante la crisis por COvID-19 en Uruguay es la politización del derecho al acompañamiento en estos procesos, derecho demandado por varias de las mujeres entrevistadas para este estudio, en consonancia con especialistas del ámbito médico y con las instituciones mencionadas.

Otro elemento emergente, no de los testimonios, sino de la situación de pandemia y del modo en que se procesa la atención médica en este contexto, se asocia con la relativización del número de controles médicos sobre el proceso de embarazo. La medicalización de los procesos de gestación, parto y puerperio ha sido ampliamente cuestionada por el feminismo, como parte de un control patriarcal sobre el cuerpo reproductivo, que tiene efectos subjetivos; en el caso de las entrevistadas, estos efectos se reflejan en las angustias ante la ausencia de un control oportuno. Pero el aumento en 21 por ciento de partos prematuros y nacidos con bajo peso, entre marzo y septiembre de 2020, en la maternidad pública del país (Gandioli, 2021), nos alerta acerca de la oportunidad política -aunque no teóricade esta relativización, teniendo en cuenta que no todas las embarazadas poseen los mismos recursos para la asistencia obstétrica y que, en condiciones excepciona- 
les como las actuales, operan otras barreras (Briozzo et al., 2020) más allá, pero en interrelación con el género, como la dificultad o retraso para identificar y valorar síntomas o motivos de consulta, y el aumento de las dificultades para el traslado o la búsqueda de atención médica. La necesidad de pensar el género en términos de interseccionalidad (Crenshaw, 2002) resulta urgente.

Por último, este trabajo evidencia el despliegue, por parte de las mujeres, de una serie de estrategias para manejar las incertidumbres derivadas de un entorno cambiante. Consecuentemente con los riesgos percibidos, todas las entrevistadas y sus redes sociales primarias adoptaron distintas medidas preventivas y estrategias de ayuda mutua, combinando la presencialidad con los intercambios mediante telefonía móvil, redes sociales y cyberactivismo. Resulta novedoso el cuidado, previo al nacimiento, de mujeres de la familia para que pudieran ayudar en las tareas de cuidado una vez en casa. Esto fue en especial relevante para las que ya tenían otros hijos / as quienes, por las medidas de cierre de los centros de enseñanza o por tener hijos demasiado pequeños, no estaban yendo a clases.

La amplitud de estrategias y prácticas preventivas adoptadas son comprendidas como parte del modelo de la autoatención definido por Menéndez (2020), y destaca en particular la dimensión relacional en el cuidado del grupo. Desde los estudios de género, remarca el carácter femenino de los cuidados, en tanto es la hermana, en el caso de Marcela, o las futuras abuelas, en los casos de Martha y Gabriela, quienes toman conductas preventivas para evitar contagiarse de la COVID- 19 y así poder estar disponibles para cuidar a las/os recién nacidas/os. En el plano analítico esto refuerza el papel central de las redes sociales primarias (sobre todo de las mujeres) en los procesos de salud-enfermedad-atención para las acciones de ayuda mutua y autoatención.

Este artículo constituye, asimismo, un aporte para el estudio de las nociones populares de riesgo y vulnerabilidad y su articulación con la determinación de las causas del proceso de salud-enfermedad-atención y las conductas de prevención, y contribuye a la comprensión de cómo la cultura atraviesa diversas prácticas y comportamientos claves en tiempos de pandemia Además, hace patente cómo las relaciones de género, en intersección con otras - de manera señalada las relaciones de clase-, imprimen formas específicas de subjetivar los procesos reproductivos, los modos de transitar el embarazo, el parto y el puerperio, y cómo imponen condiciones de atención específicas. Hemos puesto de relieve cómo, en función de una mayor capacidad económica, algunas mujeres han podido "ges- tionar" las incertidumbres relativas a los protocolos de atención cambiantes durante la pandemia por COVID-19, asegurándose una forma de atención sanitaria acorde a sus demandas, mientras otras no han tenido esta posibilidad. El trabajo evidencia también que, más allá de estas condiciones diferenciales, existen lógicas institucionales que no pueden ser desestimadas, que obligan a una adaptación por parte de las personas usuarias y que difícilmente pueden ser cuestionadas.

\section{Fuentes}

Aguirre, Rafael et al.

2020 "Recomendaciones para la mujer embarazada y el recién nacido frente a la covid-19", en $\mathrm{Mi}$ nisterio de Salud Pública, República Oriental del Uruguay, 21 de abril <https:/ /www.gub. uy/ministerio-salud-publica/comunicacion/ noticias / recomendaciones - para-mujer embarazada-recien-nacido-frente-covid-19> [28 de septiembre de 2020].

Aparicio, Matías Javier, Sofía Bilbao,

María Macarena Saenz Valenzuela

y Barán AtTias Taly Judith

2020 "Entre barbijos, ollas populares y grupos de WhatsApp: mujeres, salud y cuidados ante el COVID-19 en los barrios del Gran Buenos Aires, Argentina 2020", en Tessituras: Revista de Antropologia e Arqueologia, vol. 8, núm. 1 , pp. 279-301<https://ri.conicet.gov.ar/ handle/ 11336/120478> [ 11 de noviembre de 2020].

Arteta, ZAIDA

2020 "Declaración de la Comisión de Género del SMu en el marco de la pandemia de Covid-19", en Sindicato Médico del Uruguay (blog), 2 de abril <https: / / www.smu.org.uy/declaracionde-la-comision-de-genero-del-smu-en-elmarco-de-la-pandemia-de-covid-19/> [3 de febrero de 20201.

BARrán, José Pedro

1993 Medicina y sociedad en el Uruguay del novecientos, Tomo 1: El poder de curar, Ediciones de la Banda Oriental, Montevideo.

Barrán, José Pedro

1999 Medicina y sociedad en el Uruguay del novecientos, Tomo 3: La invención del cuerpo, Ediciones de la Banda Oriental, Montevideo.

BBC REDACCIÓN

2020 "La singular y exitosa estrategia de Uruguay para contener la pandemia de coronavirus sin cuarentena obligatoria", en BBC News Mundo (sec. América Latina), 29 de mayo <https:// www.bbc.com/mundo/noticias-americalatina-52837193> [13 de julio de 2020].

Benítez, María Alejandra,

Carolina Velasco, Ana Rita Segueira,

Josefa Henríguez, Flavio M. Menezes,

Y FRANCESCO PAOLUCCI

2020 "Responses to Covid-19 in five Latin American Countries", en Health Policy and Technology, vol. 9, núm. 4, pp. 525-559. DOI: https://doi. org/10.1016/j.hlpt.2020.08.014 [1ㅇ de diciembre de 2020]. 
BERRIO, LINA

2017 "Redes familiares y el lugar de los varones en el cuidado de la salud materna entre mujeres indígenas mexicanas", en Salud Colectiva vol. 13, núm. 3, pp. 471-87. DOI: https: / / doi. org/10.18294/sc.2017.1137 [28 de septiembre de 2020].

BRIOZZO, LEONEL (COORD.)

2002 Iniciativas sanitarias contra el aborto provocado en condiciones de riesgo, Sindicato Médico del Uruguay, Montevideo.

BRIOZZO, LEONEL ET AL.

2020 "Análisis del impacto de la pandemia covid- 19 sobre la calidad de los servicios de salud sexual y reproductiva", en Revista Médica del Uruguay, vol. 36, núm. 4, pp. 436-444. DOI: https://doi.org/10.29193/RMU.36.4.12 [2 de febrero de 2021].

ButLER, JuditH

2005 El género en disputa, Paidós, Buenos Aires.

CAstrillo, Belén

2020 "La pandemia no es excusa: parir en tiempos de derechos humanos", en Avatares de la Comunicación y la Cultura, núm. 20 <https:// publicaciones.sociales.uba.ar/index.php/ avatares/article/view/5431> [17 de diciembre de 2020].

Correa, Sonia

Y Rosalind Petchesky

1996 "Direitos Sexuais e Reprodutivos: uma perspectiva feminista", en Physis: Rev. Saúde Coletiva, vol. 6, núm. 1-2, pp. 147-177.

Costa Abós, Silvia

y MaHault Behaghel

2020 "Parir en casa en tiempos de coronavirus", en Dipòsit Digital de la Universitat de Barcelona, 30 de julio <http://diposit.ub.edu/dspace/ handle/2445/170104> [1ㅇ de octubre de 2020].

Crenshaw, Kimberlé

2002 "Documento para o encontro de especialistas em aspectos da discriminação racial relativos ao gênero", en Estudos Feministas, vol. 10, núm. 1, pp. 171-188.

DAVIS-FLOYD, RoBBIE

2001 "Los paradigmas tecnocrático, humanista y holístico del parto", en International Journal of Gynecology and Obstetrics, vol. 75, supl. 1.

DAVIS-FloYD, ROBBIE,

Kim Gutschow y David A. Schwartz

2020 "Pregnancy, Birth and the covid-19 Pandemic in the United States", en Medical Anthropology, vol. 39, núm. 5, pp. 413-427. Dor: https: / / doi.org/10.1080/01459740.2020.1761804 [ 18 de septiembre de 2020].

Esteban, Mari Luz

2006 "El estudio de la salud y el género: las ventajas de un enfoque antropológico y feminista”, en Salud Colectiva, vol. 2, núm. 1 (enero-abril), pp. 9-20.

EVIA, Victoria

2016 Etnografía en la policlínica Villa Farré: el proceso salud-enfermedad-atención desde el primer nivel, Universidad de la República Uruguay-Comisión Sectorial de Investigación Científica, Montevideo.

Freyermuth, Graciela

2003 Las mujeres de humo. Morir en Chenalhó. Género, etnia y generación, factores constitutivos del riesgo durante la maternidad, Centro de Investigaciones y Estudios Superiores en Antropología Social/Instituto Nacional de las Mujeres/Asesoría, Capacitación y Asistencia

Gainza, Patricia P. en Salud/Miguel Ángel Porrúa, México.

Mariana Labastie

y Natalia Magnone Alemán

2013 "La atención contemporánea al parto. Jaque a la perspectiva de derechos humanos", en Ulúa, núm. 22, pp. 175-196.

Gamlin, Jennie, Sahra Gibbon,

Paola Sesia y Lina Berrio (eds.)

2020 Critical Medical Anthropology in Latin America: Trends, Contributions, Possibilities, uCl Press, Londres. DOI: https: / /doi.org/10.14324/111. 9781787355828 [24 de agosto de 2020].

Gandioli, Lucía

2021 "COVID-19y consecuencias de las medidas sanitarias: nacimientos prematuros aumentaron $21 \%$ en población vulnerable entre marzo y setiembre de 2020", en La Diaria (sección Investigación científica), 2 de febrero <https: / / ladiaria.com.uy/ciencia/articulo/2021/2/ covid-19-y-consecuencias-de-las-medidassanitarias-nacimientos-prematuros aumentaron-2 1 -en-poblacion-vulnerableentre-marzo-y-setiembre-de-2020/> [2 de febrero de 2021].

ILLICH, IVÁN

1976 Medical nemesis. The expropriation of health, Pantheon Books, NuevaYork [1926].

INDDHH

2020 "Resolución 845/2020 sobre el derecho de acompañamiento en el parto en tiempos de emergencia sanitaria”, en Institución $\mathrm{Na}$ cional de Derechos Humanos y Defensoría del Pueblo <https: / /www.gub.uy/institucion nacional-derechos-humanos-uruguay/ institucional / normativa / resolucion- 8 45020-ante-denuncia-presentada-organiza cion-nacer $>$ [20 de septiembre de 2020].

Keusseian, Nicolas

2021 "Alerta por aumento de casos de embarazadas con Covid-19 graves en CTI”, en El País Uruguay, 8 de noviembre, sec. Salud $<$ https: / / www.elpais.com.uy/informacion/salud/ alerta-aumento-casos-embarazadas-covidgraves-cti.html> [8 de mayo de 2021].

LA DIARIA

2020 "Organizaciones denuncian que no se respeta el derecho de las mujeres embarazadas a estar acompañadas durante el parto o la cesárea”, en La Diaria (sección Violencias), 6 de mayo <https: / /ladiaria.com.uy/feminismos / articulo/2020/5/organizaciones-denuncianque-no-se-respeta-el-derecho-de-las-muje res-embarazadas-a-estar-acompanadasdurante-el-parto-o-la-cesarea/> [3 de septiembre de 20201.

Magnone, Natalia

2011 "Derechos y poderes en el parto: una mirada desde la perspectiva de humanización”, tesis de maestría en Trabajo Social, Facultad de Ciencias Sociales de la Universidad de la República Oriental del Uruguay.

Manderson, Lenore y Susan Levine

2020 "Covid-19, risk, fear, and fall-out", en Medical Anthropology, vol. 39, núm. 5, pp. 367-370. DOI: 
https: / / doi.org/ 10.1080/01459740.2020. 1746301

MariJanovich, Claudia

2019 "Derechos sexuales y reproductivos: parir es poder, si yo elijo", en Cotidiano Mujer <https: / / cotidianomujer.org.uy/sitio/ni-mas-nimenos/2156-derechos-sexuales-y-reproduc tivos-parir-es-poder-si-yo-elijo>.

Martin, EMiLY

1991 "The egg and the sperm: How science has constructed a romance based on stereotypical male female roles", en Signs, vol. 16, núm. 3, pp. 485-501.

Martin, EMily

2001 The Woman in the Body, Beacon Press, Boston [1987].

MEnÉndez, EduARdo L.

2009 De sujetos, saberes y estructuras: introducción al enfoque relacional en el estudio de la salud colectiva, Lugar Editorial, Buenos Aires.

MENÉndez, EduARDo L.

2020 "Acciones marginadas y ninguneadas pero básicas: coronavirus y proceso de autoatención”, en Ichan Tecolotl. La Casa del Tecolote, año 31, núm. 336, mayo <https://ichan. ciesas.edu. $\mathrm{mx} /$ acciones - marginadas $-\mathrm{y}-$ ninguneadas-pero-basicas-coronavirus-yproceso-de-autoatencion/ > [ 17 de noviembre de 2020].

Mesa, Serrana

y Mariana Viera Cherro

2004 "Tramas de significado y negociación del poder en la consulta ginecológica”, en VII Congreso Argentino de Antropología Social (CAAS), 25 al 28 de mayo de 2004, Villa Giardino, Córdoba [edición en CD ROM].

MSP

2020 "Informe epidemiológico covid-19. Actualización al 04 de diciembre de 2020", en Ministerio de Salud Pública <https: / www.gub. uy/ministerio-salud-publica/comunicacion/ noticias /informe-epidemiologico-covid-19actualizacion-04-diciembre-2020 > [16 de diciembre de 2020].

O’Connell, Maeve, Susan Crowther,

Claudia Ravaldi y Caroline Homer

2020 "Midwives in a pandemic: A call for solidarity and compassion", en Women and Birth, vol. 33, núm. 3, pp. 205-206.

OPS

2010 "Uruguay tiene la tasa de mortalidad materna más baja de América Latina”, en Organización Panamericana de la Salud/Organización Mundial de la Salud <https: / / www.paho. org/es / noticias / 8-2-2010-uruguay-tienetasa-mortalidad-materna-mas-baja-americalatina>.

OPS

2020 "COVID-19: Recomendaciones para el cuidado integral de mujeres embarazadas y recién nacidos", en Organización Panamericana de la Salud/Organización Mundial de la Salud, 27 de marzo <https://www.paho.org/clap/ images / PDF / COVID 19 embarazoyrecien nacido/COVID-19_embarazadas_y_recin nacidos_CLAP_Versin_27-03-2020.pdf? ua $=1>[2$ de febrero de 2021$]$.

Osorio, Rosa María

2011 "Construyendo puentes y abriendo caminos. La cultura médica materna como vía de aproximación a la epidemiología sociocultural", en Jaime Armando Haro (ed.), Epidemiología sociocultural. Un diálogo en torno a su sentido, métodos y alcances, Lugar Editorial, Buenos Aires.

Petruccelli, Ariel y Federico Mare

2020 "Pandemia: paranoia e hipocresía global", en Maristella Svampa et al., La fiebre. Pensamiento contemporáneo en tiempo de pandemias, Aislamiento Social Preventivo y Obligatorio (ASPO), Buenos Aires, pp. 131-168.

Robben, Antonius C. G. M.

2011 "Seducción etnográfica, transferencia, y resistencia en diálogos sobre terror y violencia en Argentina”, en Aletheia, vol. 1, núm. 2 <http://www.memoria.fahce.unlp.edu.ar/ art_revistas/pr.4824/pr.4824.pdf>.

RodRIGÁÑEZ, CASILDA

2009 Pariremos con placer. Apuntes sobre la recuperación del útero espástico y la energía sexual femenina, Madreselva, Buenos Aires.

Santana, Naiara María

2020 “'Medo do desconhecido'. Atenção às gestantes, parturientes e puérperas no contexto da COVID-19", en Boletim Cientistas sociais, núm. 29, 28 de abril.

SCOTT, JOAN

1996 "El género: Una categoría útil para el análisis histórico”, en Marta Lamas (comp.), El género: la construcción cultural de la diferencia sexual, Programa Universitario de Estudios de Género, México.

Souto, Sandra Patrícia Arantes do,

Rosemeire Sartori de Albuguergue

y Ana Paula Prata

2020 "Fear of childbirth in time of the new coronavirus pandemic", en Revista Brasileira de Enfermagem, vol. 73, supl. 2, 13 de noviembre <https: / / doi.org/10.1590/0034-7167-2020$0551>$ [ 17 de diciembre de 2020].

Vázguez-Xu, Sarah

2020 "From the epicenter, at the apex: A dispatch about birth and covid-19 from New York City", en City \& Society <https://anthrosource. onlinelibrary.wiley.com/doi / 10.1111/ciso. 12330> [ 1 o de octubre de 2020].

Zorzanelli, Rafaela Teixeira,

Francisco Ortega y Benilton Bezerra

2014 "Um panorama sobre as variações em torno do conceito de medicalização entre 19502010”, en Ciência \& Saúde Coletiva, vol. 19, núm. 6, pp. 1859-1868. 